Malgorzata Tryuk

ORCID: 0000-0002-6925-2711

Université de Varsovie

m.tryuk@uw.edu.pl

\title{
LA TRADUCTOLOGIE POLONAISE EN ANGLAIS ? DU «CENTRE » ET DE LA «PÉRIPHÉRIE » DE LA RECHERCHE TRADUCTOLOGIQUE EN POLOGNE
}

\section{INTRODUCTION}

Le titre des propos qui vont suivre peut paraître doublement frappant. D'un côté, l'objectif de mon article est de dévoiler une situation équivoque, dans le contexte du développement d'une discipline qui se veut supranationale puisqu'elle concerne le contact des cultures et des langues. Elle dépasse les frontières nationales, et par conséquent, elle englobe un public scientifique aussi large que possible. D'autre part, actuellement, l'accès aux acquis de notre discipline semble limité par le choix de la langue des publications scientifiques : elles sont habituellement en anglais. Si la nôtre est rédigée en français, c'est parce qu'elle est appelée à paraître dans Romanica Wratislaviensia, revue qui privilégie cette langue de publication. Nos propos ont toutefois l'ambition de s'adresser à une communauté de traductologues qui maîtrisent d'autres langues que l'anglais.

Après ces remarques préliminaires, rappelons les mots écrits il y a une quinzaine d'années par Yves Gambier ${ }^{1}$ :

On est [donc] témoin à la fois d'une expansion de la traductologie et d'une certaine fragmentation dans son organisation. Cette fragmentation est en partie due à des contraintes et à des motivations d'ordre linguistique et culturel. Elle est aussi en partie due aux différences dans le développement de la traductologie selon les zones géo-linguistiques. N'empêche, les défis ne sont plus exclusivement

1 Y. Gambier, « Pertinence sociale de la traductologie ? », Meta 50(4), 2005 (<https://www. erudit.org/fr/revues/meta/20050v500n40meta1024/019839ar.pdf $>$ [consulté le 29.10.2019]). 
locaux : ils se recoupent partout, que ce soit les défis de la formation, les défis de la visibilité et de la reconnaissance (accréditation), les défis de la communication multilingue, les défis des retombées de la recherche, les défis de la technologie sur les modes et les divisions du travail, etc.

Les divisions culturelles et géographiques auxquelles fait allusion Gambier résonnent dans plusieurs études réunies dans le présent volume. Ces études posent la question du centre et des périphéries, qui se reflète entre autres dans la visibilité de la recherche scientifique au niveau national, dans les retombées de celle-ci et dans son impact sur les études internationales. Or, cette visibilité repose sur l'utilisation de l'outil fondamental de communication scientifique qu'est la langue. Et cette langue est généralement l'anglais, lingua franca de la recherche en traduction et en interprétation. Cette situation et les risques liés à l'utilisation dominante de l'anglais ont déjà été relevés par Mary Snell-Hornby². Cette auteure a souligné un paradoxe : alors que les chercheurs en Translation Studies s'expriment presque exclusivement en anglais, cette discipline n'est pas la propriété exclusive de la langue anglaise. Aussi a-t-elle été la première à prôner le multilinguisme dans les débats académiques, les colloques, les publications, les citations et les bibliographies, ainsi que l'utilisation d'autres langues que l'anglais dans les travaux sur la traduction.

En Pologne, l'utilisation de la langue anglaise en traductologie a des conséquences tant pour la communication à l'intérieur de la communauté académique polonaise que pour sa visibilité dans le monde. L'anglais est utilisé comme outil de la communication dans les publications nationales, dans des thèses pour l'obtention de grades académiques, durant les colloques organisés en Pologne. C'est ainsi qu'un grand nombre de chercheurs qui n'appartiennent pas à la communauté des anglicistes ou dont la connaissance de l'anglais est limitée (mais qui, en revanche, utilisent d'autres langues étrangères comme outil de communication) peuvent se sentir exclus de l'échange scientifique, puisque leurs travaux deviennent « invisibles ».

On peut citer en guise d'exemple la situation observée dans la théorie de l'interprétation. Les années 2015-2019 ont apporté en Pologne un nombre significatif de publications dans ce domaine. En grande partie, il s'agit d'ouvrages publiés en vue de l'obtention des grades académiques de docteur ou de docteur habilité, et pris en charge par les maisons d'édition des universités polonaises ou des éditeurs étrangers ${ }^{3}$. Ils sont rédigés en anglais, et par conséquent, l'accès à ces publications

${ }^{2}$ M. Snell-Hornby, «Is Translation Studies going Anglo-Saxon? Critical Comments on the Globalization of a Discipline », [dans :] D. Gile, G. Hansen, N.K. Pokorn (dir.), Why Translation Studies Matters?, J. Benjamins, Amsterdam-Philadelphia 2010, pp. 97-104.

3 Pour n'en citer que quelques'uns : P. Gorszczyńska, Recorded Sight Translation Revisited, Gdańsk University Press, Gdańsk 2016 ; P. Korpal, Linguistic and psychological indicators of stress in simultaneous interprerting, Wydawnictwo UAM, Poznań 2017 ; K. Stachowiak-Szymczak, Eye Movements and Gestures as Correlates of Language Processing in Consecutive and Simultaneous Interpreting, Springer Verlag, Berlin 2019 ; E. Gumul Explicitation in SI. A Study into Explicitating Behaviour of Trainee Interpreters, Wydawnictwo Uniwersytetu Śląskiego, Katowice 2017 ; M. Bartłomiejczyk, Face Threats in Interpreting. A Pragmatic Study of Plenary Debates in the European Parliament, Wydawnictwo Uniwersytetu Śląskiego, Katowice 2016 ; M. Walczyński, Psycho-Affective Factors in Consecutive Interpreting, Peter Lang, Berlin 2019. 
d'une grande valeur scientifique est restreint pour une partie des chercheurs, formateurs ou professionnels de l'interprétation polonais, qui n'ont pas un niveau linguistique suffisant pour en apprécier pleinement les apports. Il semble ainsi que, par leur choix de la langue anglaise, les auteurs visent une reconnaissance extérieure, et non locale. Ils vont de la périphérie vers le centre anglo-saxon. Bien que les recherches en interprétation se portent bien dans de nombreux centres académiques, les publications en d'autres langues que l'anglais ne sont pas légion. Ainsi voit-on naître une opposition entre un «centre scientifique » qui se développe en langue anglaise et des " périphéries » qui englobent la production scientifique dans d'autres langues. Juliane House appelle cette situation « impérialisme linguistique $»^{4}$.

Il faut remarquer que cet "impérialisme » ne concerne pas seulement la traductologie ${ }^{5}$, et que les chercheurs qui décident de publier leurs résultats de recherches en anglais ne sont pas toujours libres de choisir. Ils y sont en effet souvent contraints, notamment par une volonté politique et administrative. Cette dernière prend des formes diverses, l'une - très importante - étant le classement des revues scientifiques et des éditeurs par catégories, en fonction de leur « valeur » exprimée en nombre de points attribués par les experts du Ministère de la Science et de l'Enseignement supérieur. Ces points ont a leur tour un impact sur l'évaluation des chercheurs et des établissements dans lesquels ils travaillent. Or il est de notoriété publique que les revues et les maisons d'édition les mieux « cotées » privilégient les publications en anglais.

La problématique de la publication en anglais des résultats de la recherche nationale en sciences humaines et sociales a fait l'objet d'études d'Izabela Wagner ${ }^{6}$. Selon cette sociologue, les raisons du choix de la langue anglaise dans les publications des chercheurs sont liées dans une grande mesure à des facteurs tels que l'appartenance à des réseaux scientifiques internationaux, ou encore, les paradigmes et théories de référence des auteurs.

L'objectif des remarques qui suivent est de dresser, à partir de données quantitatives, un « portrait» de la traductologie polonaise, tel qu'il surgit de l'analyse des

${ }^{4}$ J. House, «English as a lingua franca and translation », [dans :] Y. Gambier, L. van Dorsalaer (dir.), Handbook of Translation Studies, t. 4, J. Benjamins, Amsterdam-Philadelphia 2013, p. 61. Cf. aussi J. House, «English as Lingua Franca and Translation », The Translator and Interpreter Trainer 7(2), 2013, pp. 279-298.

${ }^{5}$ La problématique de la publication de textes académiques en anglais par les non natifs de cette langue a fait récemment l'objet d'un travail de Katarzyna Hryniuk, Non-natives writing for Anglo-American journals. Challenges and needs, Wydawnictwa Uniwersytetu Warszawskiego, Warszawa 2019.

${ }^{6}$ I. Wagner, « Selektywna analiza problemu publikacji humanistów i przedstawicieli nauk społecznych w języku angielskim », Przegląd Socjologii Jakościowej 8, 2012, t. 1, pp. 166-187, et eadem, «Odpowiedź na polemikę, czyli więcej na temat publikacji w języku angielskim w zagranicznych czasopismach przez polskich specjalistów nauk społecznych i humanistycznych », Przeglad Socjologii Jakościowej 8, 2012, t. 2, pp. 278-293 (<http:// www.przegladsocjologiijakosciowej.org> [consulté le 18.08.2020]). 
publications parues dans les revues internationales les mieux cotées par les autorités administratives dont dépend l'évaluation des organismes de recherche et des scientifiques polonais. Nous essaierons aussi de faire un pronostic concernant l'importance de l'anglais comme langue de publication pour la diffusion de la pensée traductologique polonaise et l'évolution possible de la recherche polonaise en traductologie.

Nous commencerons par quelques observations d'ordre général à propos de la visibilité des disciplines ou des travaux scientifiques dans le monde académique.

\section{VISIBILITÉ DES DISCIPLINES ACADÉMIQUES}

La visibilité des disciplines sur la scène internationale peut se faire de plusieurs façons. Prenons l'exemple de la traductologie.

Premièrement, la publication des ouvrages originaux de la discipline se fait dans une langue non maternelle, le plus souvent l'anglais, comme nous le montre le livre Cognitive Linguistics and Poetics of Translation d'Elżbieta Tabakowska ${ }^{7}$, qui a été traduit en polonais quelques années seulement après la parution de l'original en anglais. Un mouvement inverse se rencontre aussi, où un ouvrage important pour la discipline mais publié dans une langue de moindre diffusion est traduit quelques années après dans une langue globale. C'est par exemple le cas du livre Uměni Překladu de Jiří Levý ${ }^{8}$, paru en tchèque en 1963, sorti dans sa version allemande six ans plus tard, mais qui n'a véritablement atteint la notoriété globale qu'avec sa parution en anglais sous le titre de The Art of Translation, un demi siècle plus tard, en $2011^{9}$. Les acquis de la traductologie sont également présentés sous forme de traductions en anglais de textes majeurs de la discipline, comme cela a été le cas pour le volume Polish Translation Studies in Action. Concepts - Methodologies - Applications. A Reader ${ }^{10}$.

La propagation des idées se réalise aussi lors des colloques internationaux. Elle en est parfois même le thème principal, comme dans le cas de Going East:

${ }^{7}$ E. Tabakowska, Cognitive Linguistics and Poetics of Translation, G. Narr Verlag, Tübingen 1993 ; traduit en polonais par A. Pokojska sous le titre Językoznawstwo kognitywne a poetyka przekładu, Universitas, Kraków 2001.

8 J. Levý, Uměni Překladu, Českosklovenský spisovatel, Praha 1963 ; traduit en anglais par P. Corness sous le titre The Art of Translation, J. Benjamins, Amsterdam-Philadelphia 2011.

${ }^{9}$ Notons au passage, avec regret, que l'œuvre d'Olgierd A. Wojtasiewicz, Wstep do teorii tlumaczenia, Zakład im. Ossolińskich, Wrocław 1957, attend toujours sa version anglaise. Sur ce savant polonais, voir : M. Tryuk, « Olgierd Adrian Wojtasiewicz (1916-1995). Translator, Scholar and Trainer of Translators », [dans :] L. Schippel, C. Zwischenberger (dir.), Going East: Discovering New and Alternative Traditions in Translation Studies, Frank \& Timme, Berlin 2017, pp. 469-486.

${ }^{10}$ P. de Bończa Bukowski et M. Heydel, Polish Translation Studies in Action. Concepts Methodologies - Applications. A Reader, Peter Lang, Berlin 2019. Cet ouvrage reprend en grande partie et en traduction anglaise leur anthologie publiée quelques années auparavant en polonais (P. de Bończa Bukowski, M. Heydel, Polska myśl przekładoznawcza. Antologia, Wydawnictwo Uniwersytetu Jagiellońskiego, Kraków 2013). 
Discovering New and Alternative Traditions in Translation Studies, colloque organisé en 2015 à l'Université de Vienne ${ }^{11}$ qui a projeté une vue d'ensemble de l'évolution de la discipline en Europe centrale et orientale, dans des pays « périphériques » tels que la Pologne, la Lettonie, la Roumanie, la Russie, la Tchécoslovaquie et la République démocratique allemande.

Enfin, la visibilité des chercheurs dans une discipline peut être mesurée en fonction de leur présence dans les revues scientifiques et/ou volumes qui possèdent une résonance internationale importante auprès de la communauté académique. Or, rappelons-le, ces revues et volumes paraissent principalement en anglais.

\section{LES ÉTUDES SCIENTOMÉTRIQUES EN TRADUCTOLOGIE}

La méthode scientométrique est une application des méthodes mathématiques et statistiques aux résultats des travaux scientifiques afin de mieux comprendre les mécanismes de la recherche, considérée comme activité sociale ${ }^{12}$. Elle permet d'étudier l'évolution, la dynamique, la diffusion des idées, les directions, les tendances, parfois même les modes dans la recherche. Les disciplines académiques et les communautés scientifiques sont étudiées au macro-niveau (au niveau national ou régional), au méso-niveau (dans une université donnée, ou par un groupe particulier de chercheurs) et au micro-niveau (les travaux d'un chercheur donné) par le biais du décompte des publications, tout en prenant en considération les paramètres divers qui définissent la signification de la production scientifique dans une discipline et une zone géographique données, dans une période donnée et par rapport à la/aux langue(s) de publication. Ainsi, l'analyse des mots clés reflète l'évolution et le transfert des idées à l'intérieur d'une discipline et à travers d'autres disciplines. L'analyse des réseaux de chercheurs, des auteurs et co-auteurs, des citations permet de tracer, décrire, ou visualiser les structures, les relations entre les textes, les disciplines, les revues, les compositions des comités scientifiques des revues, les collections apportées par les maisons d'éditions, les comités scientifiques des conférences, etc. L'impact scientifique peut être mesuré également par rapport au développement de nouvelles méthodologies, aux projets de recherches ou collaborations, au transfert du savoir et des technologies, ainsi que dans la formation de nouvelles générations de chercheurs. Grâce à la méthode quantitative, on peut donc étudier l'évolution d'une discipline donnée dans une période ou une zone géographique données. En plus, elle permet de détecter les tendances, de confirmer ou d'infirmer les hypothèses scientifiques. La scientométrie est aussi un puissant outil de formation des étudiants aux études empiriques.

${ }^{11}$ L. Schippel, C. Zwischenberger (dir.), op. cit.

12 N. Grbić, " Bibliometrics », [dans :] Y. Gambier, L. van Dorslaer (dir.), Handbook of Translation Studies 4, 2013, pp. 20-24. 
En traductologie, elle est utilisée avec grand succès depuis deux décennies. Les premiers travaux datent de la fin des années 1990. Franz Pöchhacker ${ }^{13}$ et Daniel Gile $^{14}$ s'en sont servis pour démontrer l'essor considérable des travaux sur l'interprétation. Les données obtenues ont apporté des informations sur les auteurs, les écoles de pensée, les publications, la diffusion des idées par le biais des citations. Sont venues ensuite les analyses de Nadja Grbić ${ }^{15}$ à propos de l'interprétation en langue des signes, et les études de Nadja Grbić et Sonja Pöllabauer ${ }^{16}$ sur la communauté des chercheurs en interprétation communautaire et la traduction dans les pays germanophones. Ces études ont servi de modèle pour une analyse de l'état de la discipline en Espagne par les chercheuses Sara Rovira-Esteva et Pilar Orero ${ }^{17}$. Leur travail a couvert une période de trois années (de 2007 à 2009) et a porté sur le contenu de neuf revues de traductologie espagnoles. Aida Martinez-Gomez ${ }^{18}$, une autre chercheuse espagnole, a analysé le rôle des interprètes non professionnels dans l'exercice du métier. L'approche scientométrique a été appliquée à maintes reprises par des chercheurs chinois dans leurs analyses de l'état de la recherche en interprétation en Chine continentale ${ }^{19}$. Leurs travaux ont apporté des données très intéressantes sur l'émergence d'une nouvelle génération de chercheurs, les méthodologies d'étude appliquées et la richesse des sujets abordés dans la recherche. En Pologne,

13 F. Pöchhacker, « Writings and Research on Interpreting: A Bibliographic Analysis », The Interpreters'Newsletter 6, 1999, pp. 17-32. Cf. aussi F. Pöchhacker, «"Those Who Do...”: A Profile of Research(ers) in Interpreting », Target 7(1), 1999, pp. 47-64.

14 D. Gile, «The History of Research into Conference Interpreting: A Scientometric Approach », Target 12(2), 2000, pp. 297-321.

15 N. Grbić, « Where do we come from? What are we? Where are we going? A bibliometrical analysis of writings and research on sign language interpreting », The Sign Language Translator \& Interpreter 1(1), 2007, pp. 15-51.

16 N. Grbić, S Pöllabauer, « Community interpreting: Signed or spoken? Types, modes, and methods ", Linguistica Antverpiensia. New Series 5, 2006, pp. 247-261. Cf. aussi N. Grbić, S. Pöllabauer, «Counting what counts. Research on community interpreting in German speaking countries - A scientometric study », Target 20(2), 2008, pp. 297-332, et N. Grbić, S. Pöllabauer, « To count or not to count: sociometrics as a methodological tool for investigating reserach on translation and interpreting », Translation and Interpreting Studies 3(102), 2008, pp. 87-146.

17 S. Rovira-Esteva, P. Orero, « A contrastive analysis of the main benchmarking tools for research assessment in translation and interpreting: the Spanish approach », Perspectives. Studies in Translatology 19(3), 2011, pp. 233-251.

18 A. Martinez-Gomez, « Bibliometrics as a tool to map uncharted territory: A study on non-professional interpreting », Perspectives. Studies in Translatology 23(2), 2015, pp. 205-222.

19 B. Gao, M. Chai, «A Bibliometric Analysis of New Developments in Simultaneous Interpreting Studies in the West », Chinese Translators Journal 2, 2009, pp. 17-21; B. Wang, L. Mu, " Interpreter training and research in China: Recent developments », Interpreting. International Journal of Interpeting. Theory and Practice 11, 2009, pp. 267-283 ; F. Tang, « A bibliometric analysis of empirical interpreting studies in China: Based on data of experimental research papers », Foreing Language World 2, 2009, pp. 39-46 ; B. Wang, « A bibliometrical analysis of interpreting studies in China: based on a database of articles published in the CSSCI/CORE journals in recent years », Babel 61(1), 2015, pp. 62-77. 
Elżbieta Skibińska ${ }^{20}$ a analysé les références citées par les auteurs des contributions parues dans quinze volumes de la revue Między Oryginatem a Przekładem afin de dresser un aperçu des ouvrages dont s'inspirent les traductologues polonais.

En 2015, la revue Perspectives. Studies in Translation Theory and Practice a consacré un numéro à l'approche bibliométrique en traductologie. Dans ce volume, Daniel Gile ${ }^{21}$ a analysé en détail les études scientométriques réalisées jusqu'à cette date en se basant sur les données recueillies dans les publications du réseau CIRIN (Conference Interpreting Research Information Network). Les conclusions de son étude confirment l'importance de cette méthodologie dans l'observation du développement de la discipline.

\section{VISIBILITÉ DES TRADUCTOLOGUES POLONAIS. PREMIÈRE ESQUISSE}

Dans ce qui suit, nous allons utiliser une partie des données collectées pour réaliser un projet actuellement en cours, qui a pour but d'étudier la présence des traductologues polonais sur la scène éditoriale internationale. Notre analyse vient dans le prolongement d'une étude antérieure sur l'état de l'interprétologie polonaise dans les années 2004-201422.

Nous nous limitons aux publications des auteurs affiliés aux universités polonaises parues dans des revues traductologiques ou dans des volumes consacrés à la traductologie. Ainsi, nous avons pris en compte les revues figurant sur la liste ministérielle publiée en $2019^{23}$. Ensuite, nous avons analysé le contenu des volumes publiés en anglais dans les collections traductologiques des éditeurs qui figurent sur cette même liste.

Notre recherche a couvert les années 2015 à 2019, pendant lesquelles la traductologie polonaise a connu un essor considérable en raison du nombre croissant de chercheurs de la discipline et de thèses de doctorat ou d'habilitation dans le domaine ${ }^{24}$.

${ }^{20}$ E. Skibińska, «Polish Translation Studies at the Turn of Centuries: Comments from the Scientometric Perspective (On the Basis of Między Oryginatem a Przektadem [Between Original and Translation] Series) », Acta Universitatis Carolinae. Philologica 3, 2015, pp. 113-126.

${ }^{21}$ D. Gile, « Analyzing Translation studies with scientometric data: from CIRIN to citation analysis ", Perspectives. Studies in Translatology 23(2), 2015, pp. 240-248.

${ }^{22}$ M. Tryuk, " 10 ans après... L'interprétation de conférence en Pologne et en polonais », [dans :] E. Skibińska, R. Solová, K. Gostkowska (dir.), Vingt-ans après... Traduire dans une Europe en reconfiguration, Orizons, Paris 2015, pp. 335-354.

${ }^{23}$ Cf. Komunikat Ministra Nauki i Szkolnictwa Wyższego z dnia 18 grudnia 2019 r. w sprawie wykazu czasopism naukowych i recenzowanych materiałów z konferencji międzynarodowych [Communiqué du Ministre de la Science et de l'Enseignement supérieur du 18 décembre 2019 relatif à la liste des revues scientifiques et des actes de conférences internationales].

24 Notons au passage qu'en Pologne, la traductologie n'est pas considérée comme une discipline scientifique autonome ; les grades sont attribués soit en linguistique, soit en littérature. 


\section{LES REVUES DE TRADUCTOLOGIE}

Dans cette section, nous présentons les données concernant les auteurs polonais des articles parus dans des revues de traductologie qui publient uniquement en anglais (tableau 1). Ces données sont ensuite complétées par les résultats d'une exploration des revues multilingues (tableau 2). L'analyse a porté sur 15 revues internationales (10 en anglais et 5 multilingues).

\section{A. Revues publiant exclusivement en anglais}

Les revues analysées sont publiées par John Benjamins Publishing Company (Interpreting. International Journal of Research and Practice in Interpreting, Target, Translation and Interpreting Studies, Translation Spaces), le groupe britannique Taylor \& Francis (Perspectives. Studies in Translation Theory and Practice, Translation Studies, The Interpreter and Translator Trainer, The Translator) — qui inclut depuis peu la maison d'édition Routledge -, la maison d'édition hongroise Akadémiai Kiadó (Across Languages and Cultures), et l'Université d'Anvers (Linguistica Antverpiensia. New Series. Themes in Translation Studies). Pour la période analysée, nous avons répertorié 1377 articles publiés dans un total de 139 volumes. Certains titres ne publient qu'un volume par an, d'autres, de 3 à 6 volumes annuellement. Les numéros contiennent généralement entre 7 et 10 articles.

Les chercheurs polonais qui ont publié des articles ou des comptes-rendus en tant qu'auteur ou co-auteur ont été divisés en deux groupes selon leur affiliation dans les filières universitaires. Nous avons ainsi distingué les auteurs anglicistes et les non-anglicistes.

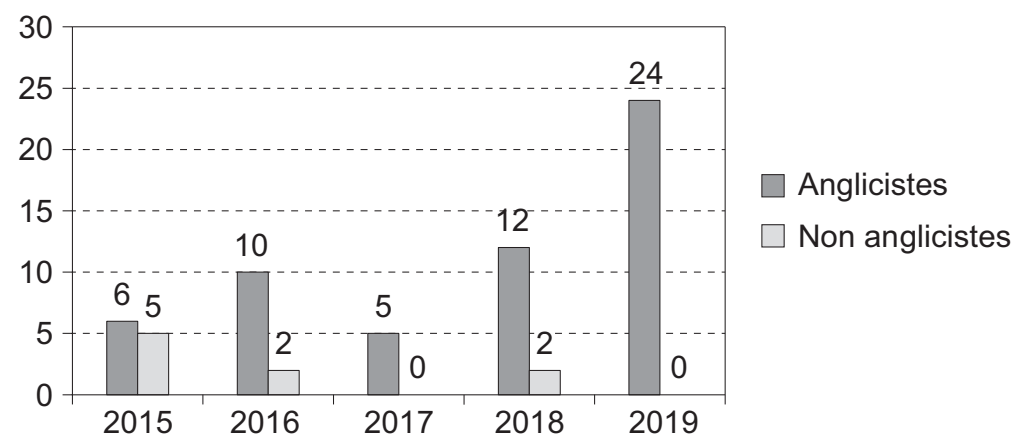

Graphique 1. L'évolution du nombre de publications d'auteurs polonais dans les revues de traductologie en anglais

Sur un total de 66 contributions de chercheurs polonais, 57 ont été publiées par des anglicistes, et les 9 autres par des auteurs non anglicistes. À la lueur de ces données, deux observations s'imposent : 
(1) Le nombre de chercheurs polonais publiant dans ces revues augmente, et c'est un signe positif de leur visibilité extérieure et peut-être aussi du développement de la discipline en Pologne. Sur la période analysée, ce nombre a doublé (11 publications au total en 2015 contre 24 en 2019). Cependant une étude détaillée révèle que dans la majorité des cas, ces chercheurs n'ont publié qu'un seul texte, et souvent en coopération avec d'autres auteurs polonais ou étrangers. L'analyse a mis au jour 37 noms d'auteurs polonais. Viennent en tête des chercheuses qui publient souvent en tant que co-auteures : Anna Kuźnik (non angliciste) avec 6 publications, Agnieszka Szarkowska, 5 textes, et Agnieszka Chmiel, 4 textes.

(2) La majorité des auteurs sont des anglicistes. Ces derniers ont publié 24 textes en 2019, contre 6 articles en 2015, alors que les non-anglicistes ont publié 9 articles, la tendance observée pour ces auteurs étant à la baisse (aucun texte en 2019). Il est probablement trop tôt pour proposer une explication de cette tendance, mais nous signalons le phénomène, il mérite certainement une observation à long terme.

\section{B. Revues multilingues}

Nous avons comparé ces données à celles des publications dans les revues de traductologie ouvertes aux auteurs publiant en d'autres langues que l'anglais. Pour la période de 2015 à 2019, nous avons répertorié au total 68 volumes et 1009 articles parus dans cinq revues (à savoir: Fachsprache. Journal of Professional and Scientific Communication, Meta, Babel, Journal of Specialised Translation, InTRAlinea; y compris les numéros spéciaux).

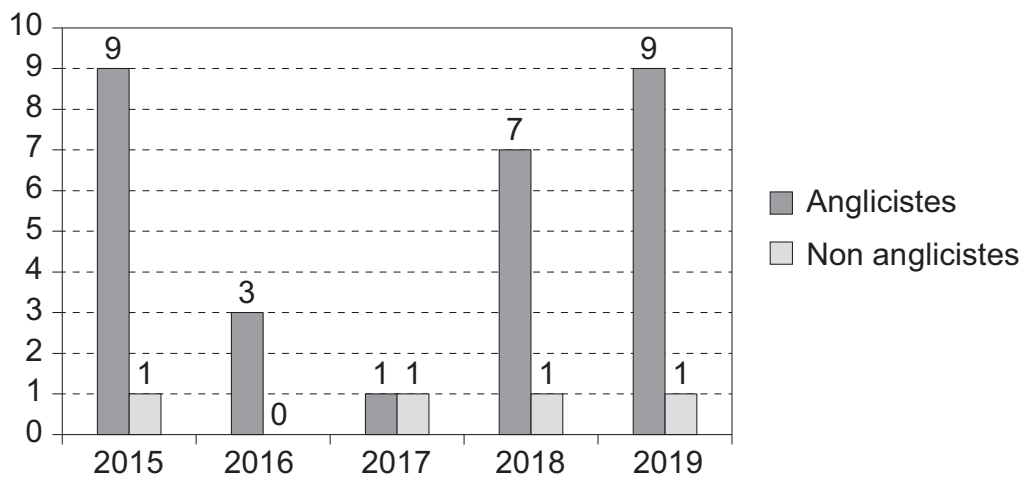

Graphique 2. L'évolution du nombre de publications d'auteurs polonais dans les revues de traductologie multilingues

Les noms d'auteurs apparaissant dans ces revues sont souvent les mêmes que dans la première série ; en majorité, ce sont des anglicistes. On y retrouve Agnieszka Szarkowska avec 5 publications, Mikołaj Deckert avec 4 publications 
et Anna Kuźnik avec 3 publications. Sur les 33 articles parus dans la période analysée, seuls 4 ont été écrits par des non-anglicistes, dont 3 dans une langue autre que l'anglais. Il s'agit d'articles en français dans la revue Meta. Là aussi, il serait intéressant d'analyser les causes de cet état des choses.

\section{LES COMMUNAUTÉS TRADUCTOLOGIQUES INTERNATIONALES}

La présence des chercheurs polonais peut se mesurer également par leur participation aux comités de rédaction des revues. Parmi les rédacteurs en chef, deux noms sont à signaler : Łucja Biel qui est la rédactrice en chef du Journal of Specialised Translation JoTRANS, et Anna Jankowska, fondatrice et rédactrice en chef de l'International Journal of Audiovisual Translation (depuis 2018).

Quatre chercheuses polonaises font partie de comités scientifiques de revues de traductologie, et toutes sont des anglicistes : Łucja Biel (Terminology. International Journal of Theoretical and Applied Issues in Specialized Communication), Bogumiła Whyatt (Translation and Translanguaging in Multilingual Contexts), Barbara Lewandowska-Tomaszczyk (Translation, Cognition \& Behaviour) et Agnieszka Szarkowska (Perspectives. Studies in Translation Theory and Practice). Cependant, il faut signaler que dans la période analysée, aucun traductologue polonais n'a publié dans Terminology ni dans Translation and Translanguaging in Multilingual Contexts. En revanche, nous avons recensé des noms d'auteurs polonais dans l'International Journal of Audiovisual Translation et dans Translation, Cognition \& Behaviour - deux revues ne figurant pas sur la liste ministérielle signalée auparavant.

\section{LIVRES DE TRADUCTOLOGIE}

1. La visibilité des traductologues polonais peut se mesurer aussi en termes de publications de volumes et de chapitres d'ouvrages publiés par les plus grandes et les plus prestigieuses maisons d'édition qui ont des collections distinctes consacrées à la recherche et à la pratique de la traduction et de l'interprétation. Nous avons analysé les publications des scientifiques polonais dans quatre maisons d'édition internationales publiant uniquement en anglais.

\section{A. John Benjamins Publishing Company}

Cet éditeur a trois collections dédiées à la recherche en traductologie. Dans ces collections, nous avons répertorié 14 noms d'auteurs de chapitres de livres parus entre 2015 et 2019. Signalons aussi que cet éditeur a publié en 2015 un volume sous la direction de deux auteurs non anglicistes. On trouve aussi chez lui une romaniste polonaise qui a contribué en tant co-éditrice à un volume publié en 2017. 
B. La maison d'édition Francis \& Taylor, qui a repris la maison Routledge, fameuse dans ce domaine, propose une série impressionnante de volumes de traductologie. Cinq traductologues polonais y ont contribué en tant qu'auteurs de chapitres et une universitaire en tant que co-éditrice de volume (en 2019).

\section{Bloomsbury}

Deux auteures anglicistes et une romaniste ont publié des chapitres dans les collections de cet éditeur dédiées à la traductologie : une en 2016 et deux en 2019.

D. Peter Lang Verlag

Cette maison d'édition suisse propose une vingtaine de collections créées à l'initiative de départements de philologie ou de linguistique des universités polonaises et qui publient des ouvrages d'auteurs polonais. Notre exploration des collections traductologiques de cet éditeur a dévoilé les noms de 34 traductologues qui ont dirigé des volumes distincts dans la période analysée. Nous y avons recensé principalement les noms des anglicistes déjà repérés dans l'analyse des revues de traductologie. Seuls deux volumes ont été publiés dans une autre langue que l'anglais, à savoir en allemand.

2. Pour terminer signalons la présence modeste des chercheurs polonais dans des encyclopédies ou des manuels (Handbook) qui ont pour ambition d'embrasser toute la connaissance à propos de la discipline. Citons les chapitres de Łucja Biel dans le Routledge Handbook of Translation Studies and Linguistics sous la direction de Kirsten Malmkjoer (2018), et de Magdalena Bartłomiejczyk, Ewa Gumul et Małgorzata Tryuk dans le Routledge Handbook of Interpreting Studies sous la direction de Franz Pöchhacker (2016).

Notre analyse témoigne d'une montée lente mais systématique de la présence des traductologues polonais dans la communauté académique internationale. Il reste à souhaiter que celle-ci se maintienne dans les années à venir.

\section{CONCLUSION}

Les données présentées ci-dessus permettent de faire de premières observations sur la présence des traductologues polonais sur la scène internationale.

1. Malgré un grand nombre de chercheurs en traductologie dans les départements et les filières de traduction polonais, leur visibilité internationale reste encore modeste. Une analyse qui reste à faire devrait révéler s'ils sont concurrencés par des auteurs d'autres régions ou pays et si cette concurrence est un obstacle à leur visibilité.

Les auteurs des publications que nous avons relevées sont dans la majorité des cas des anglicistes affiliés aux instituts ou départements de philologie anglaise des universités de Varsovie, de Łódź, de Silésie et Adam Mickiewicz 
de Poznań. Ces chercheuses et chercheurs appartiennent pour la plupart à des réseaux internationaux qui mènent des études de pointe (par ex. sur la traduction audiovisuelle ou la traductologie de corpus) dont les résultats sont souvent publiés en collaboration avec d'autres universitaires. La seule représentante non-angliciste est Anna Kuźnik, membre du groupe de recherche PACTE ${ }^{25}$ et co-auteure de nombreuses publications de ce groupe en anglais. Ainsi la participation aux projets internationaux est-elle garante d'une plus grande visibilité sur la scène internationale, ce qui a été signalé par Izabela Wagner ${ }^{26}$.

2. Nous avons vu que les anglicistes constituent le groupe d'auteurs le plus nombreux. Ceci nous amène à une réflexion sur la différence notable de situation entre eux et les chercheurs qui utilisent d'autres langues de travail. La volonté de ces derniers de publier dans les revues les mieux cotées est entravée non seulement par leur niveau d'anglais, plus faible que celui des anglicistes, mais aussi et surtout par leur manque de compétence à rédiger conformément aux conventions propres au discours académique anglo-américain, qui, notons-le au passage, sont enseignées aux étudiants d'anglais pendant leurs cours d' $"$ Academic Writing ${ }^{27}$. Pour les auteurs originaires d'autres traditions intellectuelles ou culturelles, habitués à d'autres conventions stylistiques ou textuelles, ceci constitue un défi parfois insurmontable. Le succès des publications en anglais est donc déterminé dans une grande mesure par la maîtrise du discours académique anglais. La difficulté de surmonter les habitudes formées par une autre tradition intellectuelle, disciplinaire ou culturelle, pour l'auteur qui est amené à rédiger un texte en anglais, peut expliquer que sa contribution ne passe pas l'épreuve de l'évaluation par les pairs. Pour cette raison, certains traductologues ont le sentiment d'être relégués dans une "périphérie académique » par rapport à leurs collègues anglicistes qui, eux, se trouvent au centre de la discipline.

La présente analyse ne fournit que de premières observations sur la visibilité extérieure des chercheurs polonais. Dans un deuxième temps, elle sera complétée par une analyse des données réunies dans le but de déceler les tendances et les directions de la recherche en traduction ; notamment, elle sera suivie d'une observation détaillée des sujets des articles et chapitres rédigés par les chercheurs polonais. À ce stade de notre analyse, nous avons pu constater la direction transdisciplinaire que prend actuellement la traductologie polonaise en réponse à un intérêt pour d'autres champs d'investigations (par ex. l'histoire, la sociologie, la psychologie, les sciences de la communication, etc.).

25 Groupe PACTE - Procés d'Adquisició de la Competència Traductora i Avaluació, auprès du Département de traduction et interprétation de l'Université autonome de Barcelone ( $<$ http:// grupsderecerca.uab/cat/pacte/>).

${ }^{26}$ I. Wagner, op. cit.

27 Les étudiants ont à leur disposition des manuels et guides du style académique anglo-saxon, tel le manuel de M. Piotrowska et J. Dybiec-Gajer, "Verba volant, scripta manent". How to write an M.A. Thesis in Translation Studies, Universitas, Kraków 2012. 
La question des publications co-signées mérite une analyse séparée. Nous avons déjà repéré un nombre significatif de chercheurs qui publient en tant que co-auteur avec des collègues polonais ou étrangers. Une analyse détaillée permettrait d'identifier des groupes ou des réseaux de savants travaillant autour d'un même thème.

L'analyse des citations dans les articles et volumes rédigés en anglais par les auteurs polonais appelle, elle aussi, une étude distincte susceptible d'apporter des réponses aux questions suivantes : Qui cite qui ? Qui cite les chercheurs polonais? Les auteurs en question citent-ils d'autres chercheurs polonais ? Citent-ils les travaux des auteurs polonais publiés dans d'autres langues que l'anglais ? ${ }^{28}$

Un dernier problème qui mériterait d'être soulevé dans une étude scientométrique est celui de la présence des chercheurs polonais affiliés à des universités étrangères. Nous avons repéré un nombre grandissant de traductologues, en majeure partie en poste dans les universités britanniques ou américaines, qui publient régulièrement dans les revues analysées ci-dessus. Certains ont obtenu leur grade académique dans les universités polonaises, d'autres ont été formés à l'étranger. Il serait intéressant d'analyser leur visibilité dans les publications polonaises et de voir s'ils gardent un lien avec le milieu dont ils sont originaires.

\title{
TRANSLATION STUDIES IN ENGLISH IN POLAND? ON THE CENTRE AND PERIPHERIES IN POLISH TRANSLATION STUDIES
}

\begin{abstract}
The paper focuses on the growing role of English in translation and interpreting studies in Poland and the possible consequences on communication within the Polish academic community in the country and abroad. Since more than two decades, as a rule, English has been used as a means of communication, with a growing number of publications in Polish journals and reviews, $\mathrm{PhD}$ theses defended in Polish universities and conferences held exclusively in English. As in other countries, English has become the main discussion subject in translation and interpreting studies. Consequently, a significant number of researchers who have a limited knowledge of English do not belong to the community of English studies scholars or use other languages as their means of communication and subject of research, are excluded from the scientific debate.

Using a sociometric methodology, the paper draws a profile of research and researchers based on the output of their writings and research on translation and interpreting in the years 2015-2019. It covers the evolution, the characterization of the field of research, the evolution of the Polish academic community, and finally, the dissemination of the Polish scholars' studies.

The survey ends with a reflection on possible perspectives for future sociometric analysis of translation and interpreting studies in Poland.
\end{abstract}

Key words: sociometric analysis, translation and interpreting studies, Polish scholars, journal articles, chapters.

${ }^{28}$ Sur l'intérêt d'une telle étude, voir E. Skibińska, op. cit. 